\title{
Oncogene AP2
}

National Cancer Institute

\section{Source}

National Cancer Institute. Oncogene AP2. NCI Thesaurus. Code C18331.

Human transcription factor AP-2 alpha (activating enhancer binding protein 2 alpha) (TFAP2A) gene, located at 6p24, encodes transcription factor AP-2 alpha. This gene is developmentally regulated and expression is induced by retinoic acid. Alternative splicing of the AP2 gene produces four mRNA transcripts, resulting in four protein isoforms. One of these proteins is a negative regulator of transcriptional activation by AP-2. 